\title{
Use of automated riboprinter and pulsed-field gel electrophoresis for epidemiological studies of invasive Haemophilus influenzae in Taiwan
}

\author{
CHIH-CHIEN WANG, L.K. SIU*, MIN-KUNG CHEN†, YEN-LING YU‡, F. M. LIN*, MONTO HO* and \\ MONG-LING CHU
}

Department of Paediatrics, Tri-Service General Hospital, National Defense Medical Center, * Division of Clinical Research, National Health Research Institute, †Department of Paediatrics, Taichung Provincial Hospital and $\$$ Center for Diseases Control, Department of Health, Taiwan

\begin{abstract}
A total of 87 invasive isolates of Haemophilus influenzae isolated throughout Taiwan from 1994 to 1998 was collected; 57 were from children $<14$ years old. In all, $60.9 \%$ of isolates were resistant to ampicillin and produced $\beta$-lactamase. Ribotyping revealed six different profiles in 55 isolates of type b, nine profiles in 10 isolates of non-type b and 12 profiles in 22 isolates of non-typable $H$. influenzae. Among isolates from 35 cases of meningitis, $30(86 \%)$ were in ribogroups 1,2 and 3 with $>90 \%$ genetic similarity. Compared with all the other ribogroups, ribogroups 1,2 and 3 , which encompassed all $H$. influenzae type $b$, were significantly more prevalent as a cause of meningitis in children $<14$ years old. Further subtyping of the predominant ribogroup by pulsed-field gel electrophoresis (PFGE) identified differences of 0-6 bands among these isolates of ribogroup 1, which indicated distant relatedness. Automated ribotyping was found to be a useful method and was less time-consuming for molecular epidemiology studies of $\boldsymbol{H}$. influenzae. PFGE is suggested as an addition to ribotyping to improve discrimination if $H$. influenzae type $b$ is involved. Differentiating ribogroups between type $b$ and non-type b $\boldsymbol{H}$. influenzae by genotyping may help to understand the molecular characteristics of outbreaks, endemicity and value of vaccination. According to the results of ribotyping and PFGE, it seems possible that spread of invasive $H$. influenzae type b had occurred and ribotyping confirmed that there was no clonal spread of non-type $b$ H. influenzae in Taiwan.
\end{abstract}

\section{Introduction}

Haemophilus influenzae is an important cause of morbidity and mortality causing pneumonia, meningitis and other invasive infections among infants and young children [1-3]. Although the incidence of $H$. influenzae type b (Hib) disease has dropped dramatically since the conjugate $\mathrm{Hib}$ vaccine was introduced in the USA [4], there are no comparable data from vaccine studies in Taiwan. Furthermore, Hib disease continues to occur either because vaccination has not been strictly enforced or there is non-type $b$ infection. The clinical characteristics of invasive $H$. influenzae disease among populations of different ages vary widely. Data on the clinical significance of both invasive Hib and non-Hib $H$. influenzae in Taiwan, and the epidemiology of the

Received 1 Feb. 2000; revised version received 11 Aug. 2000; accepted 23 Aug. 2000.

Corresponding author: Dr M-L. Chu (e-mail: mlchu@cde. org.tw) disease, are limited. A previous study showed that traditional ribotyping with EcoRI-digested total DNA and cDNA probes for $16 \mathrm{~S}$ and $23 \mathrm{~S}$ rRNA-encoding genes is a highly discriminatory method for $H$. influenzae [5,6]. In addition to ribotyping, pulsed-field gel electrophoresis (PFGE) has also been suggested by other groups [5, 7-9]. However, these methods involve lengthy procedures of cell wall lysis, DNA extraction, restriction endonuclease digestion and $>20 \mathrm{~h}$ of gel separation, and blotting, hybridisation and detection steps are required for ribotyping. Thus, it often takes up to a week to obtain results. For these reasons, automated computerised ribotyping and PFGE were used to characterise invasive $H$. influenzae isolates in Taiwan to investigate their epidemiology.

Materials and methods

Bacterial isolates

Clinical isolates of invasive $H$. influenzae were 
obtained from hospitals throughout Taiwan. Pertinent information including case history, clinical course, significant sequelae and prognoses was collected in a uniform manner for analysis. After identification in the local hospital, isolates were sent to the central laboratory in the Division of Bacterial Diseases, Center for Diseases Control (CDC), Department of Health in Taipei. The presence or absence of capsular polysaccharide as detected by the Bacto $H$. influenzae antisera (Difco, Detroit, MI, USA) separated typable from non-typable isolates. Typable isolates were serotyped by the slide agglutination test with type $b$ antisera (Difco) to further separate them into type $b$ and non-type b. Susceptibility to ampicillin was determined by a disk diffusion test, which was interpreted according to NCCLS criteria [10]. $\beta$ Lactamase screening was performed with a nitrocefin disk (BBL, Cockeysville, MD, USA).

\section{Automated ribotyping}

Ribotyping was performed with the automated Riboprinter ${ }^{\mathrm{TM}}$ Microbial Characterization System (Qualicon, Wilmington, DE, USA) according to the manufacturer's instructions. Colonies were picked and loaded into the Riboprinter ${ }^{\mathrm{TM}}$ Microbial Characterization Unit (MCU). Within the MCU, total DNA was digested with EcoRI and DNA was separated by electrophoresis and transferred directly to nylon membranes. Ribopatterns were expressed by hybridisation with a chemiluminscent-labelled DNA probe containing an rRNA operon (rrnB) from Escherichia coli. The patterns were automatically imaged and stored in the MCU computer. The positions of standard markers were used to correct for lane-to-lane and membrane-to-membrane variations in band position. The ribopattern for each isolate was compared to other patterns in the Riboprinter ${ }^{\mathrm{TM}}$ database. Assignment to a particular ribogroup is based upon differences in band numbers, band position and signal intensity at a given banding position [11].

\section{PFGE}

Total DNA was prepared and PFGE was performed as described previously [12]. The restriction endonuclease Sma I (Biolabs, Beverly, MA, USA) was used at the manufacturer's suggested temperature. Restriction fragments were separated by PFGE in agarose $1 \%$ gel (BioRad, Hercules, CA, USA) in $0.5 \times \mathrm{TBE}$ buffer (45 mM Tris, $45 \mathrm{~mm}$ boric acid, $1.0 \mathrm{~mm}$ EDTA, $\mathrm{pH}$ 8.0) with the BioRad CHEF Mapper apparatus. The initial pulse time of $1 \mathrm{~s}$ was increased linearly to $35 \mathrm{~s}$ over $28.4 \mathrm{~h}$ at $200 \mathrm{~V}$ at $14^{\circ} \mathrm{C}$. Gels were then stained with ethidium bromide and photographed under ultraviolet light.

Band patterns were compared visually and classified as indistinguishable (clonal), closely related (clonal variants, three or fewer band differences), possibly related (four-to-six band differences) and unrelated (over six band differences) according to previously described criteria [13]. Genetic similarity was calculated by the unweighted pair group method using arithmetic averages (UPGMA) and shown in a dendrogram. Similarity was adjusted by Jaccard's coefficients with tolerance $0.5 \%$. The program for calculation was supplied by BioRad.

\section{Results}

\section{Bacterial isolates}

In all, 87 isolates of invasive $H$. influenzae were collected and identified in the Division of Bacterial Diseases, CDC; all were from cerebrospinal fluid, blood or pleural fluid. Demographic information indicated that 45 patients $(51.7 \%)$ resided in Northern Taiwan (Taipei 30, Keelung 1 and Taoyan 14), 13 patients $(15 \%)$ in Central Taiwan (Taichung 10 and Changhua 3), 24 patients $(27 \%)$ in Southern Taiwan (Chiayi 1, Tainan 3 and Kaohsiung 20) and 5 patients (6\%) in Eastern Taiwan (Hualien 5) (Fig. 1). Serotyping revealed 55 isolates $(63 \%)$ to be type b, $10(12 \%)$ non-type $\mathrm{b}$ and $22(25 \%)$ non-typable $H$. influenzae. Fifty-three isolates (61\%) were ampicillin resistant and all the resistant isolates produced a $\beta$-lactamase (Table $1)$.

\section{Automated ribotyping}

Molecular study by automated ribotyping yielded 23 profiles among the 89 isolates (including two ATCC strains, 49247 and 49766). Six different profiles were identified in 55 type b isolates, 9 profiles in 10 nontype $\mathrm{b}$ isolates and 12 profiles in 22 non-typable isolates of $H$. influenzae. There were four major profiles among the type $b$ isolates and two major profiles among the non-typable isolates (Table 2). The dendrogram shown in Fig. 2 depicts the overall similarities between different ribotypes. Ribogroup 1, which included the ATCC 49247 control strain, group 2 and group 3 showed $>90 \%$ similarity to each other. Type $\mathrm{b} H$. influenzae was the main constituent of ribogroups 1 and 2. The ribotype profile of ATCC strain 49766 was different from those of the 87 invasive isolates and was classified as ribogroup 21. Other than ribogroups 1, 2 and 3, non-type b and nontypable isolates were highly variable (patterns 4-23) in ribopattern.

Fifty-seven cases of invasive $H$. influenzae disease were identified in children $<14$ years of age; $42(74 \%)$ of these belonged to ribogroups 1, 2 and 3 (Table 3 ). Among isolates identified as belonging to ribogroup 1, 2, 3 and 6 in children, all were found to be Hib with different clinical presentations. There were 35 cases of meningitis and these were associated with ribogroups 1 , 2, 3, 6 and 9; $22(63 \%)$ cases of meningitis were associated with ribogroup 1 and four cases each with groups 2, 3 and 6. Ribogroup 9 comprised the only 


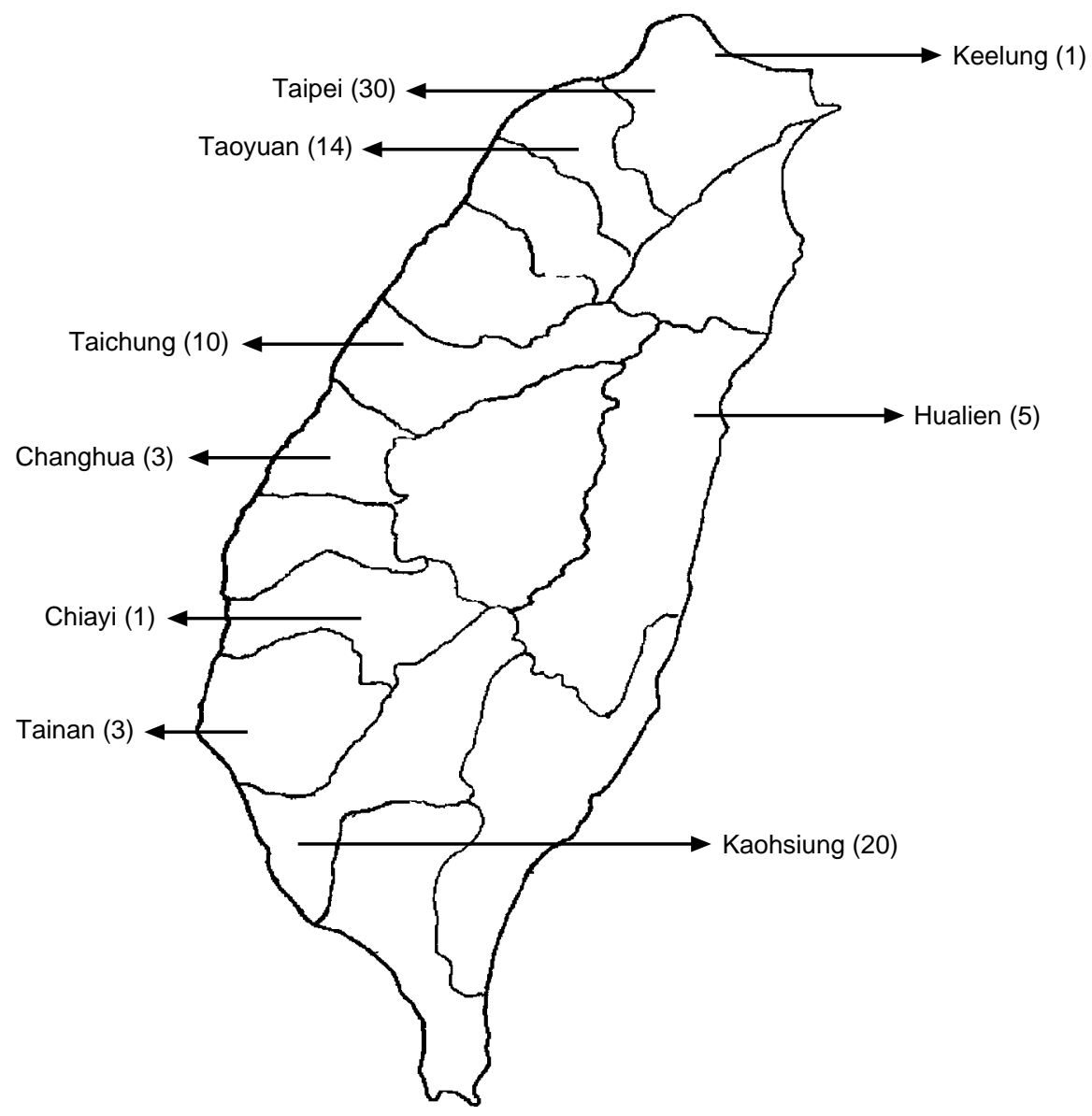

Fig. 1. Geographic distribution of invasive H. influenzae in this study.

Table 1. Characteristics of invasive $H$. influenzae isolates, 1994-1998

\begin{tabular}{|c|c|c|c|c|}
\hline \multirow[b]{2}{*}{ Year } & \multicolumn{2}{|c|}{ Number of isolates } & \multirow{2}{*}{$\begin{array}{l}\text { Ampicillin } \\
\text { resitance (\%) }\end{array}$} & \multirow{2}{*}{$\begin{array}{c}\beta \text {-Lactamase } \\
\text { test* }(\%)\end{array}$} \\
\hline & Type $b$ & Others & & \\
\hline 1994 & 1 & 2 & $2(66.7)$ & $2(66.7)$ \\
\hline 1995 & 3 & 2 & $4(80.0)$ & $4(80.0)$ \\
\hline 1996 & 24 & 22 & $24(52.2)$ & $24(52.2)$ \\
\hline 1997 & 19 & 6 & $17(68.0)$ & $17(68.0)$ \\
\hline 1998 & 8 & 0 & $6(75)$ & $6(75)$ \\
\hline Total & 55 & 32 & $53(60.9)$ & $53(60.9)$ \\
\hline
\end{tabular}

*Ampicillin-resistant isolates that were positive in the nitrocefin disk test.

non-type $\mathrm{b} H$. influenzae isolate that caused meningitis and was the only isolate classified in this group. Compared with all the other ribogroups, ribogroups 1, 2 and 3 which were closely related $(>90 \%$ genetic similarity) were significantly more prevalent (30 of 35 cases) as causes of meningitis in children under 14 years old.

PFGE of isolates from paediatric patients with meningitis caused by ribogroup $1 \mathrm{H}$. influenzae

In subtyping ribogroup 1 isolates by PFGE, 20 subtypes were identified with $0-6$ band differences (Fig. 3). According to the interpretative criteria for PFGE, all these isolates in ribogroup 1 are clonal, clonally related or possibly related. There were no specific subtype differences found among the isolates associated with meningitis, sepsis, pneumonia, cellulitis or epiglottitis.

\section{Discussion}

$H$. influenzae is still one of the most important causes of infection in children, including sepsis, pneumonia and meningitis. Non-capsulate strains can colonise the upper respiratory tract and may be asymptomatic or cause mucosal infections such as otitis media, sinusitis, bronchitis and conjunctivitis.

Recently, PFGE has become recognised as a useful method for molecular typing and has been used for typing $H$. influenzae serotype b isolates [14-16]. Furthermore, Gazagne et al. found that PFGE, APPCR with two sets of primers and ribotyping created nearly the same number of patterns in a study of clinical ampicillin-resistant, non- $\beta$-lactamase-producing H. influenzae [5]. Although 23 profiles were identified by ribotyping and AP-PCR and 20 profiles were identified by PFGE, Gazagne et al. claimed that ribotyping was more complicated and time-consuming than the other two methods. Thus, ribotyping was not recommended as a tool for molecular typing of $H$. influenzae [5]. 
Table 2. Ribogroup patterns of invasive $H$. influenzae isolates of different serotypes

\begin{tabular}{|c|c|c|c|c|c|}
\hline Ribogroup & Type b & Non-type $b$ & Non-typable & Control strains* & Total \\
\hline 1 & 33 & 2 & & 1 & 36 \\
\hline 2 & 6 & & & & 6 \\
\hline 3 & 8 & 1 & 1 & & 10 \\
\hline 4 & & 1 & 5 & & 6 \\
\hline 5 & 2 & & & & 2 \\
\hline 6 & 5 & & 1 & & 6 \\
\hline 7 & & & 1 & & 1 \\
\hline 8 & & & 1 & & 1 \\
\hline 9 & & & 7 & & 7 \\
\hline 10 & & 1 & & & 1 \\
\hline 11 & & 1 & & & 1 \\
\hline 12 & & 1 & & & 1 \\
\hline 13 & & 1 & & & 1 \\
\hline 14 & & & 1 & & 1 \\
\hline 15 & & 1 & & & 1 \\
\hline 16 & & & 1 & & 1 \\
\hline 17 & & 1 & & & 1 \\
\hline 18 & & & 1 & & 1 \\
\hline 19 & & & 1 & & 1 \\
\hline 20 & & & 1 & & 1 \\
\hline 21 & & & & 1 & 1 \\
\hline 22 & 1 & & & & 1 \\
\hline 23 & & & 1 & & 1 \\
\hline Total & 55 & 10 & 22 & 2 & 89 \\
\hline
\end{tabular}

*ATCC 49247 and 49766.

Similarity (\%)

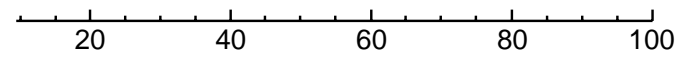

Number

of isolates $\quad b \quad$ non-b non-typable
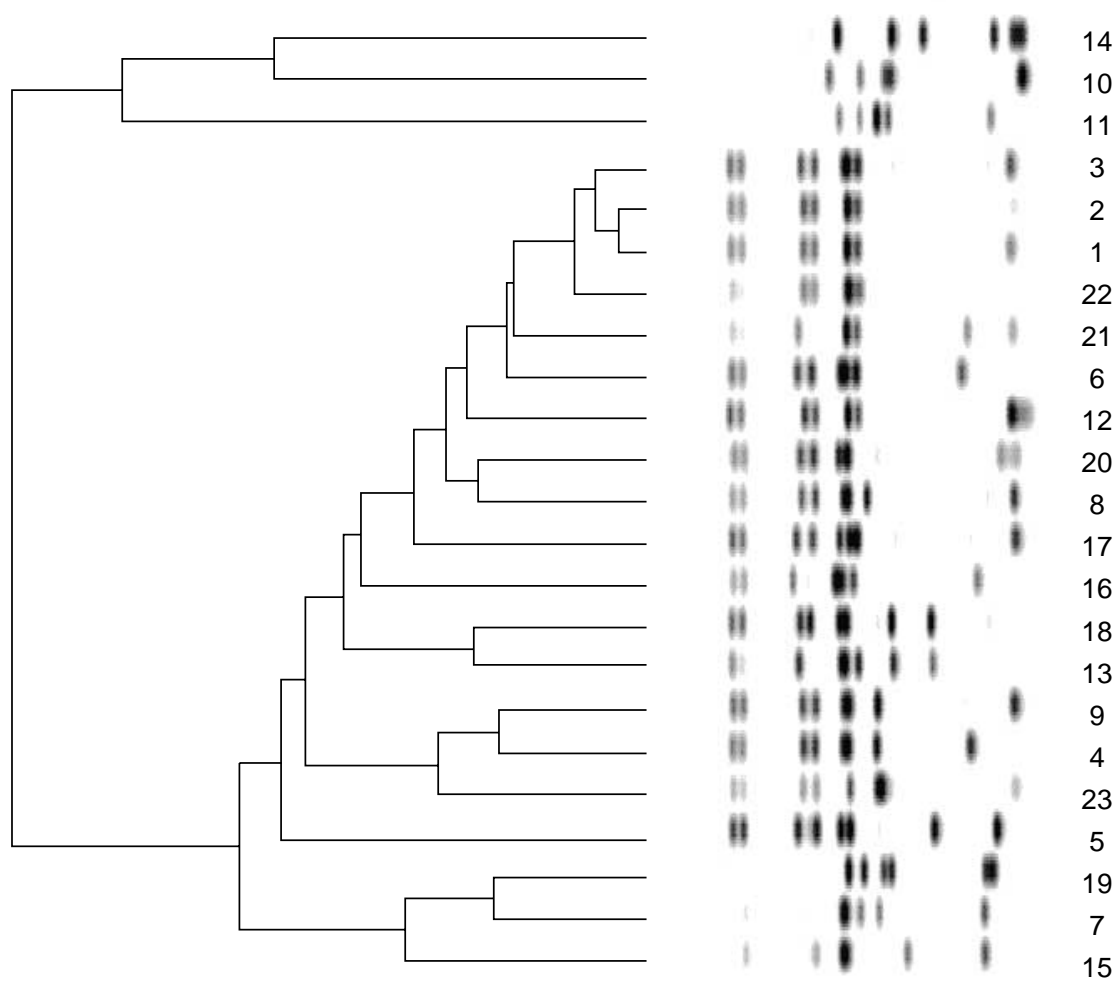

Fig. 2. Riboprofiles and dendrogram computer-generated by automated riboprinter of $87 \mathrm{H}$. influenzae clinical isolates and ATCC control strains 49247 and 49766. A similarity matrix (upper right) was calculated by the UPGMA clustering method with a global optimisation option ( $1 \%$ jitter compensation).

One major limitation of molecular typing methods is lack of standardisation. The results cannot be electronically transferable between laboratories and the information is difficult to store in the computer for future follow-up studies and comparison with previous studies. This variability has created uncertainty regarding the usefulness of the various methods. One approach to the development of standardised methods is to provide 
Table 3. Association between ribogroups and $H$. influenzae disease in children $<14$ years old

\begin{tabular}{|c|c|c|c|c|c|c|c|c|c|c|}
\hline \multirow[b]{2}{*}{ Ribogroup } & \multicolumn{2}{|c|}{ Meningitis } & \multicolumn{2}{|c|}{ Sepsis } & \multicolumn{2}{|c|}{ Pneumonia } & \multicolumn{2}{|c|}{ Cellulitis } & \multicolumn{2}{|c|}{ Epiglottitis } \\
\hline & Type b & Others* & Type b & Others & Type b & Others & Type b & Others & Type b & Others \\
\hline 1 & 22 & & 2 & & 2 & & 2 & & 1 & \\
\hline 2 & 4 & & & & 2 & & & & & \\
\hline 3 & 4 & & 1 & & 2 & & & & & \\
\hline 4 & & & & 2 & & & & & & \\
\hline 5 & & & & & 1 & & & & & \\
\hline 6 & 4 & & 1 & & & & & & & \\
\hline 8 & & & & 1 & & & & & & \\
\hline 9 & & 1 & & & & & & & & \\
\hline 12 & & & & & & 1 & & & & \\
\hline 15 & & & & 1 & & & & & & \\
\hline 17 & & & & 1 & & & & & & \\
\hline 18 & & & & & & 1 & & & & \\
\hline 23 & & & 1 & & & & & & & \\
\hline
\end{tabular}

* Others refers to either non-type b or non-typable $H$. influenzae.

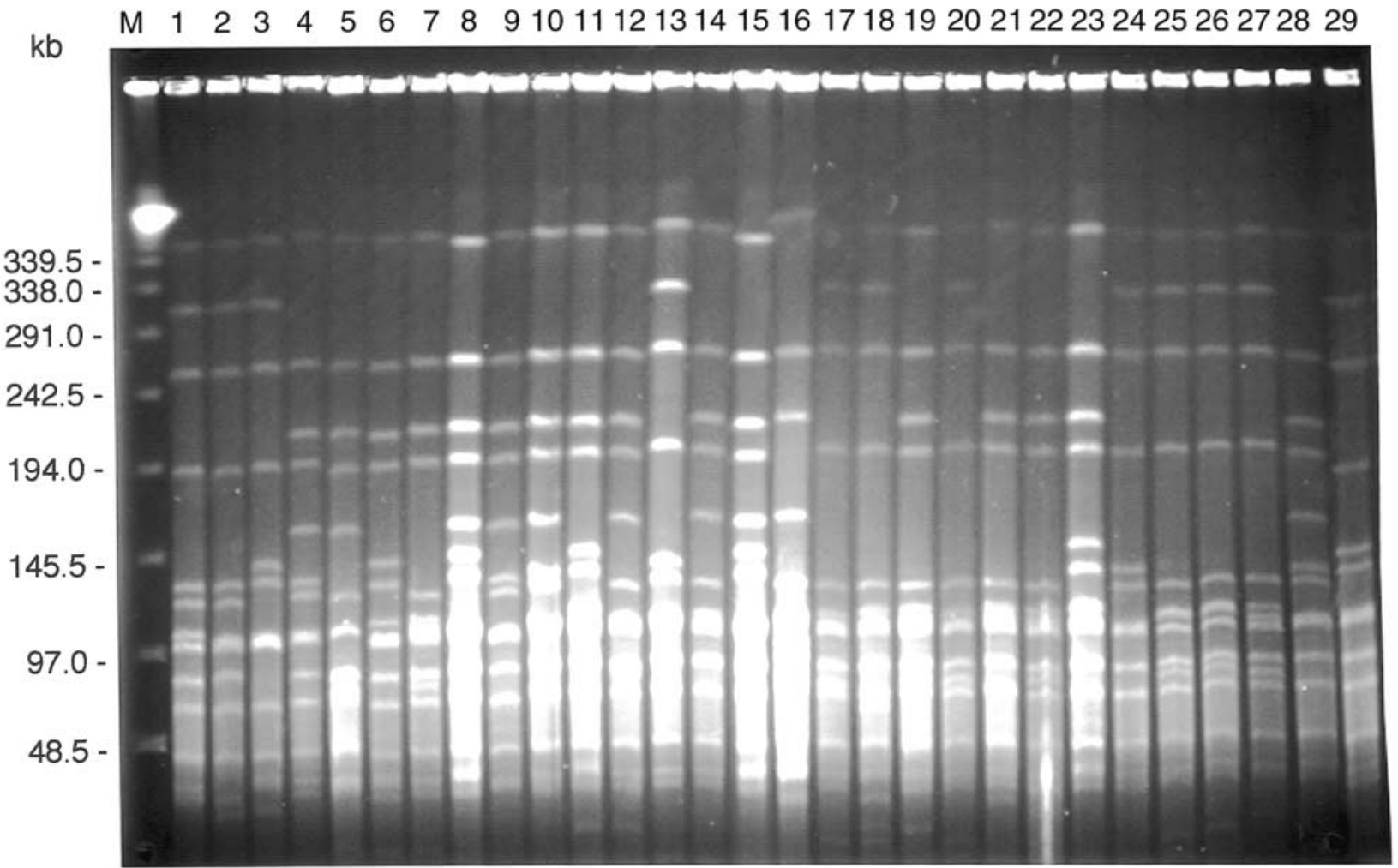

Fig. 3. PFGE patterns of isolates of ribogroup 1 for children aged $<14$ years old. The isolates were from meningitis (lanes 1-22), sepsis (23 and 24), pneumonia (25 and 26), cellulitis (27 and 28) and epiglottitis (29).

an automated kit-based assay $[17,18]$. Such an approach not only standardises the technical and interpretive aspects of a procedure, but also uses a computer database to compare profiles from large numbers of isolates. Thus, an automated ribotyping system in which results can be obtained within $8 \mathrm{~h}$ for 16 isolates was used initially in the present study. This was followed by further study with PFGE when a predominant ribogroup was obtained. As there is no previous information on epidemiological studies of $H$. influenzae by automated ribotyping, the discriminatory power of ribotyping cannot be assessed if a predominant ribogroup is obtained. Thus PFGE was used to further assess the discriminatory power of the automated ribotyping in this study.

In this study, a total of 87 invasive $H$. influenzae isolates was collected for epidemiological study. Among them, 46 isolates (52.9\%) were from Northern Taiwan. As most of the academic medical centres are currently located in Northern Taiwan and $74 \%$ of all $H$. influenzae isolates were collected from academic medical centres, the relatively larger number of isolates from this region was not unexpected.

This study showed that the prevalence of ampicillin- 
resistant $H$. influenzae in Taiwan is very high (61\%) and $67 \%$ of $H$. influenzae type b (Hib) isolates from children with meningitis produced $\beta$-lactamase. However, the rate of ampicillin resistance in this study was similar to that in a previous study in 1994-1995 [18]. An intriguing finding was that all the ampicillinresistant strains produced $\beta$-lactamase. Although $\beta$ lactamase production is the major mechanism of resistance found in $H$. influenzae, it accounts for only $20 \%$ of resistance in France, Belgium and Spain, 25\% in Mexico, 28\% in Saudi Arabia, 37\% in Hong Kong and $30 \%$ in the USA $[19,20]$.

Analysis of the riboprinter patterns in the present study showed that most of the Hib isolates were of the same clone or closely related subclones. According to the results of ribotyping (Table 3), clonal propagation of invasive $H$. influenzae has apparently occurred in Taiwan. Further subtyping by PFGE revealed that isolates of ribogroup 1 from 22 cases of meningitis and 7 cases of other infections in children had $0-6$ different bands. With reference to the interpretation of PFGE, all these strains should be considered distantly related. Thus, the combination of ribotyping and PFGE might be required to ascribe clonality of Hib.

Compared with all the other ribogroups, ribogroups 1 , 2 and 3 were significantly more prevalent as a cause of meningitis in children $<14$ years old. As all isolates in ribogroups 1, 2 and 3 were Hib (Table 3), the results obtained with ribotyping were comparable to previous studies showing that $\mathrm{Hib}$ is the major cause of meningitis. Tarasi et al. demonstrated by PFGE that non-epidemiologically related Hib strains comprised a major endemic clone with a number of subclones. Thus, they suggested that the population structure of capsulate $H$. influenzae is basically clonal and that most infections are caused by a small number of clonal genotypes. The results obtained in the present study support the previous findings [21,22]. Different riboprinter patterns were obtained for non-type $b$ and non-typable $H$. influenzae isolates. The non-typable $H$. influenzae isolates showed riboprinter patterns that appeared to be distinct from each other and unrelated to those of Hib isolates. This is in line with previous studies by multilocus enzyme electrophoresis [23] and PFGE [5] showing that non-typable $H$. influenzae are genetically heterogeneous and belong to clones different from Hib. Furthermore, the present study found that the non-type b capsulate $H$. influenzae isolates could also be differentiated by ribotyping. This finding may be useful in the future for discriminating type $b$, nontype $b$ and non-typable strains. As virulence in nontype $\mathrm{b}$ and non-typable strains can be transferable between $H$. influenzae strains [24], monitoring of these should be continued after initiation of the vaccination program to explain vaccine failures.

In conclusion, specific ribogroups of $H$. influenzae with high levels of genetic relatedness were identified as major causes of meningitis in children. As ribotyping was found to be a useful method for molecular epidemiological studies for both Hib and non-Hib and was comparable to PFGE but less time-consuming, future use of this method for world-wide or regional studies can enhance understanding of the routes of transmission of type $\mathrm{b}$, non-type $\mathrm{b}$ and non-typable $H$. influenzae infections.

We are grateful to Ms Ching-Mei $\mathrm{Yu}$ for her technical assistance. This research was supported by grants from the National Science Council, Executive Yuan, Taiwan (NSC-88-2314-B016-026) and the National Health Research Institute, Taiwan.

\section{References}

1. Levine OS, Schwartz B. The rationale for population-based surveillance for Haemophilus influenzae type b meningitis. Pediatr Infect Dis J 1998; 17 Suppl 9: S195-S198.

2. Schuchat A, Robinson K, Wenger JD et al. Bacterial meningitis in the United States in 1995. N Engl J Med 1997; 337: 970-976.

3. Wang $\mathrm{CH}$, Lin TY. Invasive Haemophilus influenzae diseases and purulent meningitis in Taiwan. J Formos Med Assoc 1996; 95: 599-604.

4. Adams WG, Deaver KA, Cochi SL et al. Decline of childhood Haemophilus influenzae type $\mathrm{b}$ (Hib) disease in the Hib vaccine era. JAMA 1993; 269: 221-226.

5. Gazagne L, Delmas C, Bingen E, Dabernat H. Molecular epidemiology of ampicillin-resistant non-beta-lactamase-producing Haemophilus influenzae. J Clin Microbiol 1998; 36: 3629-3635.

6. Jordens JZ, Leaves NI. Source of variation detected in ribotyping patterns of Haemophilus influenzae: comparison of traditional ribotyping, PCR-ribotyping and rDNA restriction analysis. J Med Microbiol 1997; 46: 763-772.

7. Aparicio P, Roman F, Campos J. [Epidemiological characterization of Haemophilus influenzae using molecular markers.] Enferm Infecc Microbiol Clin 1996; 14: 227-232.

8. Moor PE, Collignon PC, Gilbert GL. Pulsed-field gel electrophoresis used to investigate genetic diversity of Haemophilus influenzae type $\mathrm{b}$ isolates in Australia shows differences between Aboriginal and non-Aboriginal isolates. J Clin Microbiol 1999; 37: 1524-1531.

9. Saito M, Umeda A, Yoshida SI. Subtyping of Haemophilus influenzae strains by pulsed-field gel electrophoresis. J Clin Microbiol 1999; 37: 2142-2147.

10. National Committee for Clinical Laboratory Standards. Performance standards for antimicrobial disk susceptibility tests: Approved standard M2-A6. Villanova, PA, National Committee for Clinical Laboratory Standards. 1997.

11. Pfaller MA, Wendt C, Hollis RJ et al. Comparative evaluation of an automated ribotyping system versus pulsed-field gel electrophoresis for epidemiological typing of clinical isolates of Escherichia coli and Pseudomonas aeruginosa from patients with recurrent gram-negative bacteremia. Diagn Microbiol Infect Dis 1996; 25: 1-8.

12. Schoonmaker D, Heimberger T, Birkhead G. Comparison of ribotyping and restriction enzyme analysis using pulse-field gel electrophoresis for distinguishing Legionella pneumophila isolates obtained during a nosocomial outbreak. $J$ Clin Microbiol 1992; 30: 1491-1498.

13. Tenover FC, Arbeit RD, Goering RV et al. Interpreting chromosomal DNA restriction patterns produced by pulsedfield gel electrophoresis: criteria for bacterial strain typing. J Clin Microbiol 1995; 33: 2233-2239.

14. Heath TC, Hewitt MC, Jalaludin B et al. Invasive Haemophilus influenzae type $\mathrm{b}$ disease in elderly nursing home residents: two related cases. Emerg Infect Dis 1997; 3: 179-182.

15. Mitsuda T, Kuroki H, Ishikawa N et al. Molecular epidemiological study of Haemophilus influenzae serotype b strains obtained from children with meningitis in Japan. $J$ Clin Microbiol 1999; 37: 2548-2552. 
16. Inzana TJ, Pichichero ME. Lipopolysaccharide subtypes of Haemophilus influenzae type $\mathrm{b}$ from an outbreak of invasive disease. J Clin Microbiol 1984; 20: 145-150.

17. Vos P, Hogers R, Bleeker M et al. AFLP: a new technique for DNA fingerprinting. Nucleic Acids Res 1995; 23: 4407-4414.

18. Lin $\mathrm{HC}$, Wang $\mathrm{CC}, \mathrm{Yu} \mathrm{CM}$, Chu ML. Prevalence of antimicrobial resistance among clinical isolates of Haemophilus influenzae in Taiwan. J Formos Med Assoc 1999; 98: 319-325.

19. Felmingham D, Washington J. Trends in the antimicrobial susceptibility of bacterial respiratory tract pathogens - findings of the Alexander Project 1992-1996. J Chemother 1999; 11 Suppl 1: 5-21.

20. Felmingham D, Grüneberg $\mathrm{RN}$ and The Alexander Project Group. A multicentre collaborative study of the antimicrobial susceptibility of community-acquired, lower respiratory tract pathogens 1992-1993: The Alexander Project. J Antimicrob
Chemother 1996; 38 Suppl A: 1-57.

21. Musser JM, Barenkamp SJ, Granoff DM, Selander RK. Genetic relationships of serologically nontypable and serotype b strains of Haemophilus influenzae. Infect Immun 1986; 52: 183-191.

22. Tarasi A, D'Ambrosio F, Perrone G, Pantosti A. Susceptibility and genetic relatedness of invasive Haemophilus influenzae type b in Italy. Microb Drug Resist 1998; 4: 301-306.

23. Murphy TF, Bernstein JM, Dryja DM, Campagnari AA, Apicella MA. Outer membrane protein and lipooligosaccharide analysis of paired nasopharyngeal and middle ear isolates in otitis media due to nontypable Haemophilus influenzae: pathogenetic and epidemiological observations. $J$ Infect Dis 1987; 156: 723-731.

24. Kroll JS, Booy R. Haemophilus influenzae: capsule vaccine and capsulation genetics. Mol Med Today 1996; 2: 160165. 